\title{
Morfeas con manifestaciones cefálicas
}

\section{Morpheas with cephalic manifestations}

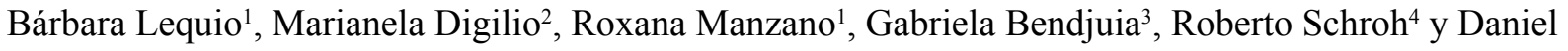
Feinsilber $^{5}$

\section{RESUMEN}

Introducción: La hemiatrofia facial progresiva (HFP) o síndrome de Parry-Romberg y la morfea en golpe de sable (MGS) forman parte de las morfeas lineales cefálicas. Son enfermedades inflamatorias crónicas de la piel y tejidos subyacentes, que se caracterizan por esclerosis y atrofia cutánea.

Objetivos: Describir las características clínicas, manifestaciones asociadas, histología, laboratorio, estudios complementarios y tratamientos instaurados.

Diseño: Estudio retrospectivo descriptivo.

Materiales y métodos: Se revisaron las historias clínicas de pacientes con morfea evaluados en el Sector Colagenopatías desde julio de 2010 hasta diciembre de 2016.
Resultados: De 56 pacientes, 11 cumplieron con los criterios de inclusión, 7 con diagnóstico de HFP, 2 con MGS y 2 con ambas patologías. El $64 \%$ fueron mujeres. Las manifestaciones extracutáneas se presentaron en el $64 \%$ de los casos. El tratamiento utilizado en todos los pacientes fue el metotrexato, asociado o no, al uso de corticosteroides sistémicos.

Conclusiones: La mayoría de nuestros resultados concuerdan con la bibliografía consultada, excepto las manifestaciones asociadas. Destacamos el tratamiento asociado de metotrexato y pulsos de corticosteroides intravenosos con resultados satisfactorios y bien tolerado.

Palabras clave: Morfea lineal, morfea en golpe de sable, hemiatrofia facial progresiva, síndrome de Parry-Romberg.

Dermatol. Argent. 2020, 26 (1): 11-16

\section{ABSTRACT}

Introduction: Progressive facial hemiatrophy (PFH) or Parry-Romberg Syndrome and morphea en coup de sabre are cephalic linear morpheas. They are chronics inflammatories diseases of the skin and underlying tissues, characterized by cutaneous atrophy and sclerosis.

Objectives: To describe clinical features, associated extracutaneous manifestations, histological and laboratory findings, imaging and diagnostic modalities and treatments established in patients with diagnosis of HFP, MGS, or both, evaluated in our Department.

Design: Retrospective descriptive study.

Materials and methods: We included medical histories of patients diagnosed with morphea evaluated in Collagenopathy Sector from July 2010 up to December 2016.
Results: Of 56 patients, 11 met the inclusion criteria, 7 with diagnosis of PFH, 2 with morphea en coup de sabre and 2 with both pathologies. $64 \%$ were women. $64 \%$ showed extracutaneous manifestations. The treatment used in all of the patients was methotrexate, associated or not, with the use of systemic corticosteroids.

Conclusions: Most of our results agree with the bibliography consulted, with the exception of the associated manifestations. We emphasize the associated treatment of methotrexate and intravenous corticosteroid pulses with satisfactory results and well tolerated.

Keys words: linear morphea, morphea en coup de sabre, progressive facial hemiatrophy, Parry-Romberg Syndrome

Dermatol. Argent. 2020, 26 (1): 11-16

\footnotetext{
${ }^{1}$ Médica Dermatóloga. Ex Médica Concurrente

${ }^{2}$ Médica Dermatóloga. Ex Jefa de Residentes

${ }^{3}$ Médica Dermatóloga. Médica de Planta, Sector Colagenopatías

${ }^{4}$ Médico Dermatólogo y Patólogo. Ex Médico de Planta, Servicio de Anatomía Patológica

5 Jefe de División, Servicio de Dermatología Hospital General de Agudos J. M. Ramos Mejía, Ciudad Autónoma de Buenos Aires, Argentina
}

Contacto del autor: Bárbara Lequio

E-mail: barbaralequio@gmail.com

Fecha de trabajo recibido: 16/7/2019

Fecha de trabajo aceptado: 12/12/2019

Conflicto de interés: los autores declaran que no existe conflicto de interés. 


\section{INTRODUCCIÓN}

La hemiatrofia facial progresiva (HFP), también llamada síndrome de Parry-Romberg, es una entidad de etiología desconocida, caracterizada por la aparición lenta y progresiva de lesiones atróficas que comprometen de forma unilateral la piel de la cara. Comienza con máculas hipopigmentadas o hiperpigmentadas, ligeramente induradas, que progresan hasta generar atrofia de la piel y los tejidos subyacentes. Se localiza principalmente en los dermatomas de una o más ramas del nervio trigémino $(\mathrm{V} \text { par })^{1}$.

La morfea en golpe de sable (MGS) se manifiesta con engrosamiento y endurecimiento cutáneo, de disposición lineal, que afecta la cara y/o el cuero cabelludo. Al igual que la HFP, puede generar atrofia de los músculos y huesos subyacentes y provocar alteraciones estéticas y funcionales².

Algunos pacientes presentan lesiones de ambas entidades simultáneamente o progresión de una entidad a otra, por lo que varios autores proponen que son variantes clínicas de la misma enfermedad ${ }^{2}$.

\section{OBJETIVOS}

Describir las características clínicas, las manifestaciones asociadas, los hallazgos histológicos y de laboratorio, los estudios complementarios y los tratamientos instaurados en los pacientes evaluados en nuestro Servicio con diagnóstico de HFP, MGS o ambas entidades.

\section{MATERIALES Y MÉTODOS}

Se realizó un estudio retrospectivo y descriptivo para el cual se revisaron todas las historias clínicas de pacientes con diagnóstico de morfea evaluados en el Sector de Colagenopatías desde el 1 de julio de 2010 hasta el 31 diciembre de 2016.

Se incluyeron todos los pacientes con morfea en el polo cefálico con diagnóstico clínico o histopatológico, asociada o no a placas extracefálicas. Las variables analizadas fueron: sexo, edad, antecedentes personales, características clínicas y localización de las lesiones, manifestaciones extracutáneas, alteraciones de laboratorio, estudios complementarios, histología y tratamiento.

\section{RESULTADOS}

De los 56 pacientes con diagnóstico de morfea, $11(20 \%)$ cumplieron con los criterios de inclusión al presentar morfea a nivel del polo cefálico. Los $11 \mathrm{pa}-$ cientes seleccionados con diagnóstico de HFP, MGS o ambas patologías, tenían entre 17 y 56 años. El 64\% (7 de 11) fueron mujeres. Del total, 7 tenían HFP, 2 MGS y los 2 restantes presentaron simultáneamente ambas entidades: HFP + MGS.
Los antecedentes de relevancia fueron: tiroiditis de Hashimoto en una paciente con HFP, múltiples traumatismos craneoencefálicos sin pérdida de conocimiento en la niñez de un paciente y epilepsia en otro paciente, ambos con MGS.

Respecto de las manifestaciones clínicas, los pacientes con diagnóstico de HFP presentaron atrofia en el $100 \%$ de los casos, induración solo en uno de ellos y discromías en 5 de 7 pacientes (4 hiperpigmentación y 1 hipopigmentación). La región más afectada de la cara resultó la inervada por la rama mandibular del nervio trigémino, observada en $43 \%$ de los pacientes ( 3 de 7 ), otro $28,5 \%$ ( 2 de 7 ) presentaron afectación de toda la hemicara y, finalmente, otro $28,5 \%$ ( 2 de 7 ) con compromiso de la rama maxilar superior (Fotos 1 y 2 ).

Los diagnosticados como MGS (2 de 11) presentaron la lesión a nivel frontoparietal. Aquellos con diagnóstico de ambas patologías en forma concomitante ( 2 de 11 ) presentaron la lesión en golpe de sable a nivel frontal, asociada, en uno de los casos, a afección de la región maxilar superior del trigémino (Foto 3) y en el otro, a afección de toda una hemicara, ambas homolaterales a la MGS.

Se documentó la presencia de otras placas de morfea extracefálica en 3 de 11 pacientes. Esta asociación se observó en 2 mujeres con HFP que presentaron una placa circunscripta en el abdomen y en el glúteo, respectivamente, y otra en una mujer con diagnóstico HFP + 
F0T02: Hemiatrofia facial progresiva que compromete la región V3, asociada a espasmos masticatorios.

MGS que presentaba dos placas de morfea circunscripta profunda en el dorso. Todas las placas de morfea extracefálicas resultaron homolaterales a la lesión cefálica.

Las manifestaciones extracutáneas las presentó el 64\% (7 de 11) de los pacientes. Las alteraciones odontológicas y maxilofaciales (espasmos masticatorios y compromiso lingual, uvular o dental) se observaron en un $71 \%$ de los pacientes ( 5 de 7 ), las alteraciones neurológicas (cefaleas y parestesias) en otro $71 \%(5 \mathrm{de} 7)$ y las oftalmológicas (enoftalmia) en un $28 \%$ (2 de 7 ). El síntoma aislado más frecuente fue la cefalea en un 57\% de los pacientes ( 4 de 7). Los pacientes con MGS exclusivo no presentaron manifestaciones extracutáneas asociadas (Gráfico 1 y Tabla 1).

Entre los hallazgos de laboratorio, 3 pacientes presentaron valores de eritrosedimentación acelerada, 1 presentó eosinofilia y otro, creatina fosfocinasa (CPK) elevada. Además, se observó ANA positivo, título $1 / 320$, en un solo paciente.

Otros estudios complementarios realizados en el $100 \%$ de los casos, para estudiar las probables manifestaciones asociadas, fueron: tomografía axial computada (TC) y/o resonancia magnética nuclear (RM) de cerebro y macizo facial, evaluación oftalmológica, otorrinolaringológica y odontológica. Los hallazgos en los estudios por imágenes se observaron en tres de los pacientes con HFP y fueron: una calcificación cerebral aislada en un paciente, áreas glioticas subcorticales en otro y displasia fibrosa ósea
FOT0 3: Hemiatrofia facial progresiva que compromete la región V2 y morfea en golpe de sable.

cráneo facial en el restante. Se realizó ecografía de piel y partes blandas de la zona afectada y en forma comparativa con el lado sano contralateral, a modo de control y seguimiento. Este estudio se realizó en tres pacientes y se evidenció estabilización de las lesiones.

El tratamiento utilizado en todos los pacientes fue el metotrexato, asociado o no al uso de corticosteroides sistémicos por vía oral en forma diaria (2 de 11) o en pulsos intravenosos mensuales ( 6 de 11), con buena respuesta y sin progresión de la enfermedad. Tres de las pacientes con HFP, luego de 2 años de estabilizadas sus lesiones, recibieron trasplante de grasa autóloga, con buenos resultados estéticos.

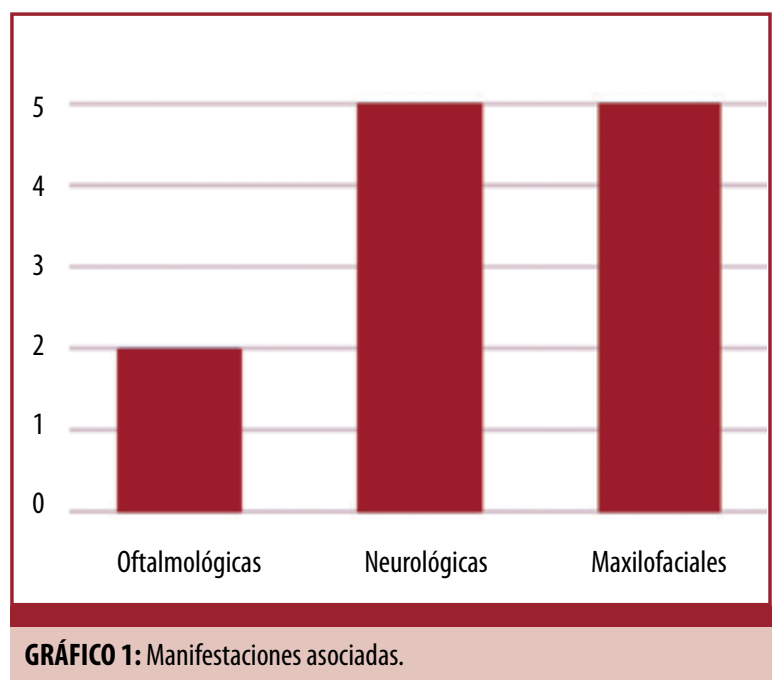




\begin{tabular}{|c|c|c|c|c|c|c|c|c|c|c|c|c|}
\hline Paciente & & 1 & 2 & 3 & 4 & 5 & 6 & 7 & 8 & 9 & 10 & 11 \\
\hline Edad & & 18 & 31 & 51 & 56 & 17 & 17 & 35 & 16 & 17 & 29 & 18 \\
\hline Sexo & & $\mathrm{F}$ & $\mathrm{F}$ & $\mathrm{F}$ & $\mathrm{F}$ & $\mathrm{F}$ & $\mathrm{F}$ & $\mathrm{F}$ & M & M & M & M \\
\hline HFP & & $x$ & & $X$ & $x$ & $x$ & & $X$ & & $x$ & $x$ & \\
\hline MGS & & & & & & & & & $x$ & & & $x$ \\
\hline HFP + MGS & & & $x$ & & & & $x$ & & & & & \\
\hline Placas extracefálicas & & & & $x$ & & $x$ & $x$ & & & & & \\
\hline \multirow{3}{*}{ Síntomas asociados } & Neurológicas & $x$ & & $x$ & & $x$ & $x$ & & & & $x$ & \\
\hline & $\begin{array}{l}\text { Maxilofaciales y } \\
\text { odontológicas }\end{array}$ & & & $x$ & & $x$ & $x$ & & & $x$ & $x$ & \\
\hline & Oftalmológicas & & & & & $x$ & & & & $x$ & & \\
\hline
\end{tabular}

\section{DISCUSIÓN}

En 2006, Laxer y Zulian clasificaron las morfeas en cinco grupos: circunscripta, lineal, generalizada, panesclerótica y mixta ${ }^{3}$. Las morfeas lineales se subdividen en: morfeas del tronco y las extremidades y morfeas de la cabeza que, a su vez, se las subclasifica en: hemiatrofia facial progresiva o síndrome de $\mathrm{Pa}$ rry-Romberg y morfea en golpe de sable ${ }^{1,3}$.

Las morfeas lineales son el subtipo más frecuente de morfeas en los niños y, dentro de estas, las más prevalentes son la MGS, la HFP y la morfea lineal que compromete las extremidades ${ }^{4}$. La HFP y la MGS se observan con mayor frecuencia en los niños, en personas jóvenes y en mujeres caucásicas, con una relación 2-4:1 con respecto al hombre ${ }^{1}$. La edad de presentación de la mayoría de nuestros pacientes estaba en las primeras dos décadas de la vida, al igual que lo descripto en la bibliografía, y el $64 \%$ eran mujeres.

La etiología se desconoce, pero se describieron factores genéticos, alteraciones vasculares y traumatismos como factores activadores o iniciadores de la enfer$\operatorname{medad}^{4}$. Debido a la gran coincidencia que hay entre las morfeas lineales y las líneas de Blashko, se sugiere que parte de su mecanismo patogénico se debe a un mosaicismo genético ${ }^{1,4}$. Su asociación a enfermedades autoinmunes y el hallazgo de títulos altos de anticuerpos antinucleares (ANA) sustentan la hipótesis de que se trata de un mecanismo autoinmune ${ }^{5}$. En nuestra casuística, un paciente tenía tiroiditis de Hashimoto; otro, epilepsia, y un tercero refirió múltiples traumatismos craneoencefálicos en su infancia.

Clínicamente, se observa una lesión unilateral deprimida, atrófica, que puede ser hiperpigmentada o hipopigmentada, a veces indurada, de superficie lisa y brillante ${ }^{6}$. No hay criterios para distinguir una MGS de una HFP, pero sí se pueden observar ciertas características clínicas para identificarlas. La disposición lineal e indurada en la región frontoparietal sugiere una MGS, mientras que una lesión clínicamente atrófica, hiperpigmentada o hipopigmentada, localizada topográficamente en la región de inervación de las ramas (V1, V2, V3) del nervio trigémino, sugiere una $\mathrm{HFP}^{2}$. En nuestro estudio, se observó con mayor frecuencia la forma de presentación como HFP (7 de 11): solo 2 pacientes presentaron MGS. Ambas formas clínicas pueden coexistir en un $20-37 \%$ de los casos, situación detectada en 2 de nuestros pacientes ${ }^{1,2}$.

Además, un $30-40 \%$ de los pacientes con HFP pueden presentar lesiones de morfea a nivel extrafacial $^{1}$. Tres de nuestros pacientes con HFP presentaron también placas escleroatróficas extracefálicas, por lo que se clasificaron como morfeas mixtas.

Las morfeas localizadas en el polo cefálico pueden acompañarse de manifestaciones neurológicas en un $20 \%$, oftalmológicas en un $15 \%$, maxilofaciales y odontológicas, lo que obliga a realizar un estudio y un abordaje multidisciplinario ${ }^{1,6}$. Las manifestaciones neurológicas más frecuentes son epilepsia, migrańa, neuralgia, parestesias de pares craneales, anomalías en el electroencefalograma y hallazgos en las neuroimágenes $^{6,7}$. Pueden hallarse calcificaciones subcorticales, hamartomas o atrofia cerebral en la RM. En su mayoría permanecen asintomáticos ${ }^{8}$. Nuestros pacientes presentaron cefalea, parestesias y uno refirió antecedentes de epilepsia. Tres de los pacientes con HFP presentaron alteraciones en los estudios por imágenes (TC y RM): calcificación cerebral aislada, áreas glióticas subcorticales y displasia fibrosa ósea craneofacial, todos hallazgos descriptos como posibles asociaciones 
de la HFP. Con respecto a las manifestaciones oftalmológicas, predominan la esclerosis de las estructuras anexiales, la inflamación del segmento anterior y la uveítis anterior, pero también se describen la parálisis de la musculatura ocular, la ptosis, el síndrome de Horner y el enoftalmos ${ }^{6}$. Las manifestaciones maxilofaciales y odontológicas más frecuentes son deformaciones de la mandíbula con desviación de la comisura, maloclusión dental, implantación inadecuada de los dientes, atrofia de las raíces, espasmos masticatorios y alteraciones de la lengua o de la úvula ${ }^{6}$.

En el laboratorio, puede haber eosinofilia y eritrosedimentación (VSG) acelerada en casos de enfermedad activa y CPK elevada, cuando existe miositis subyacente $^{1,8}$. En nuestra casuística, 3 pacientes presentaron VSG acelerada, uno solo eosinofilia y otro, CPK elevada. Los ANA, con patrón homogéneo y moteado, se encuentran positivos en el 37-50\% de los pacientes con morfeas lineales y no se correlacionan con la actividad de la enfermedad ${ }^{1}$. En nuestro estudio, un paciente tenía ANA positivo, con valor de $1 / 320$. Si bien ciertos autoanticuerpos (anti-DNA, antihistona, antitopoisomerasa II alfa, antirribonucleoproteína, anticuerpos antimetaloproteinasas, entre otros) se observaron en algunos pacientes, no se recomienda su tamizaje ${ }^{8}$.

La ecografía de la piel y las partes blandas aporta datos objetivos y sensibles acerca de la afectación, profundidad y cambios del compromiso cutáneo?. Es útil para evaluar el grado de afectación en forma comparativa con la piel sana. El estudio debe realizarse al inicio y al final del tratamiento, idealmente por el mismo operador y sobre un área representativa de la afectación cutánea ${ }^{10}$.

Las características histopatológicas de las morfeas se evidencian principalmente en la dermis y la hipodermis. Es posible observar el reemplazo de distintas estructuras por bandas fibrocolágenas, marcada atrofia dérmica y engrosamiento de los vasos rodeados por infiltrado linfoplasmocitario. En los estadios más avanzados, se observa la atrofoesclerodermia y, en algunos casos, atrofia dérmica sin esclerosis ${ }^{1,8,11}$. En nuestro estudio, 2 de los pacientes con MGS presentaron atrofoesclerodermia, mientras que de los pacientes con HFP, 4 presentaron atrofoesclerodermia y 3 , atrofia dérmica sin esclerosis.

El curso evolutivo de la enfermedad es lento. La progresión puede ser entre 2 y 20 años hasta estabilizarse $^{1}$. El riesgo de que una morfea lineal evolucione a esclerosis sistémica es de 0,9 a $1,3 \%{ }^{1}$. Un único paciente de nuestro estudio presentó fenómeno de Raynaud, capilaroscopía normal y un ANA de $1 / 320$; quien continúa en estudio y seguimiento. Cabe destacar que el fenómeno de Raynaud se considera un factor de riesgo independiente para esta posible evolución ${ }^{1}$.

La terapéutica se elige según la extensión y gravedad de la enfermedad con el objetivo de detener la actividad y evitar las secuelas. No existe un consen$\mathrm{so}^{12}$. Algunos estudios sugieren como primera línea el metotrexato (15-25 mg semanales) asociado a prednisona por vía oral $(0,5$ a $1 \mathrm{mg} / \mathrm{kg} /$ día $)$ o a pulsos mensuales de metilprednisolona por vía intravenosa $(0,5$ a $1 \mathrm{~g} /$ día durante 3 días consecutivos por 3 a 6 meses $)^{7,13}$. Esta última vía de administración se prefiere debido a su menor riesgo de sufrir efectos adversos $^{1,6}$. El objetivo es combinar el efecto antiinflamatorio de los corticosteroides con el efecto antifibrótico del metotrexato ${ }^{6}$. En nuestra casuística, todos los pacientes recibieron metotrexato durante un tiempo prolongado, con buena tolerancia. De estos, en solo 2 se asociaron corticosteroides orales y 6 fueron tratados en forma combinada con pulsos intravenosos de metilprednisolona. Como segunda opción terapéutica se encuentra el micofenolato de mofetilo $y$, en tercer lugar, la fototerapia PUVA, UVA1 y UVB banda angosta ${ }^{6,8,9}$.

El seguimiento es fundamentalmente mediante la evaluación clínica ${ }^{4}$. Con fines de investigación, se utiliza el Modified Skin Score (MSS) o el Localized Scleroderma Cutaneous Assessment Tool (LoSCAT) $)^{5,8}$.

\section{CONCLUSIONES}

Nuestro estudio y la bibliografía consultada permiten destacar que tanto la MGS como la HFP son más frecuentes en las primeras dos décadas de la vida. En esta etapa de crecimiento, si no se comienza un tratamiento oportuno, la enfermedad evoluciona con secuelas irreversibles. De ahí la importancia de realizar un abordaje multidisciplinario, detectar precozmente las manifestaciones asociadas y efectuar un correcto seguimiento.

$\mathrm{Si}$ bien las manifestaciones neurológicas y oftalmológicas aparecen como las asociaciones más frecuentes en la bibliografía publicada hasta el momento, la mayoría de nuestros pacientes presentaron alteraciones maxilofaciales y odontológicas. Esto puede deberse al mayor compromiso del territorio inervado por la rama mandibular del trigémino de los pacientes estudiados.

El mecanismo fisiopatológico de las morfeas es complejo y, al no haber diferencias histológicas entre una HFP y una MGS, creemos que ambas forman parte del espectro de una misma entidad clínica. 


\section{BIBLIOGRAFÍA}

1. Figueiroa Careta M, Romiti R. Localized scleroderma: clinical spectrum and therapeutic update. An Bras Dermatol 2015;90:62-73

2. El-Kehdy J, Abbas O, Rubeiz N. A review of Parry-Romberg syndrome. J Am Acad Dermatol 2012;67:769-784.

3. Laxer LM, Zulian F. Localized scleroderma. Curr Opin Rheumatol 2006;18:606-613.

4. Fett N, Werth V. Update on Morphea Part I. Epidemiology, clinical presentation, and pathogenesis. J Am Acad Dermatol 2011;64: 217-228.

5. Zulian F, Athreya BH, Laxer R, Nelson AM, et ál. Juvenile localized scleroderma: clinical and epidemiological features in 750 children. An international study. Rheumatology (Oxford) 2006;45:614-620.

6. Bielsa Marsol I. Actualización en la clasificación y el tratamiento de la esclerodermia localizada. Actas Dermosifiliogr 2013;104:654-666.

7. Knobler R, Moinzadeh P, Hunzelmann N, Kreuter A, et ál. European Dermatology Forum S1-guideline on the diagnosis and treatment of sclerosing diseases of the skin, Part 1: localized scleroderma, systemic sclerosis and overlap síndromes. J Eur Acad Dermatol Venereol 2017;31:1401-1424.
8. Fett N, Werth V. Update on Morphea Part II. Outcome measures and treatment. J Am Acad Dermatol 2011;64:231242.

9. Kreuter A, Gambichler T, Breuckmann F, Rotterdam S, et ál Pulsed high-dose corticosteroids combined with low-dose methotrexate in severe localized scleroderma. Arch Dermatol 2005; 141:847-852.

10. Buján M, Merediz J, Nogales M, Cervini AB, et ál. Esclerodermia lineal en "coup de sabre" y síndrome de Parry-Romberg. Estudio retrospectivo en un hospital pediátrico. Arch Argent Dermatol 2009;59:43-52.

11. Zulian F, Vallongo C, Patrizi A, Belloni-Fortina A, et ál. A longterm follow-up study of methotrexate in juvenile localized scleroderma (morphea). J Am Acad Dermatol 2012;67:11511156.

12. Zwischenberger BA, Jacobe HT. A systematic review of morphea treatments and therapeutic algorithm. J Am Acad Dermatol 2011;65:925-941.

13. Sommer A, Gambichler T, Bacharach-Buhles $M$, Von Rothenburg T, et ál. Clinical and serological characteristics of progressive facial hemiatrophy: a case series of 12 patients. $J$ Am Acad Dermatol 2006;54:227-233.

\section{PERLAS}

Federico Pastore

Médico Dermatólogo y Legista. Médico de Planta de Dermatología del Hospital Dr. E. Tornú

Jefe del Servicio de Dermatología del Instituto César Milstein.

\section{PSORIASIS Y VITAMINA D}

La psoriasis es una enfermedad

inflamatoria crónica mediada inmunológicamente, de base genética, que se puede desencadenar 0 agravar por diferentes factores. La 25-hidroxivitamina D (25[OH] D) tiene efectos antiproliferativos, antiinflamatorios y antiangiogénicos en el organismo.

En un metanálisis en el que se incluyeron 571 pacientes con psoriasis y 496 personas sanas, se midieron los niveles plasmáticos de $25(\mathrm{OH}) \mathrm{D}$ en ambos grupos, la severidad de la enfermedad en los pacientes con el Psoriasis Area Severity Index (PASI) y la relación entre la gravedad de la psoriasis y el nivel de deficiencia de la vitamina.

El metanálisis concluyó que los niveles circulantes de $25(\mathrm{OH}) \mathrm{D}$ son más bajos en los pacientes con psoriasis y que existe una correlación estadísticamente signifi- cativa entre esos niveles y la gravedad de la psoriasis.

Lee YH, Song GG. Association between circulating 25-hydroxyvitamin D levels and psoriasis, and correlation with disease severity: a meta-analysis. Clin Exp Dermatol 2018;43:529-535.

\section{SEGURIDAD EN EL USO DE ANTIHISTA- MÍNICOS DE SEGUNDA GENERACIÓN EN LA URTICARIA CRÓNICA}

Los antihistamínicos de segunda generación son el tratamiento de primera línea contra la urticaria crónica. Un porcentaje de pacientes no responden a las dosis utilizadas habitualmente de estos medicamentos. Las guías EAACI/GA2 LEN/EDF/WAO sugieren, en estos casos, aumentar la dosis hasta cuadruplicarlas. Estas últimas son dosis offlabel (fuera de prospecto) y la responsabilidad de la indicación es del médico prescriptor, por lo que el perfil de seguridad del medi- camento es de vital importancia cuando se utiliza por encima de las dosis autorizadas. Debe valorarse la potencial cardiotoxicidad en este grupo de medicamentos, dado que pueden prolongar el intervalo QT.

Este estudio concluyó que el perfil de seguridad cardiovascular de los antihistamínicos usados en el tratamiento de la urticaria crónica, aun en dosis cuatro veces mayores que las habituales, es aceptable. Sin embargo, debe tenerse especial precaución en los pacientes con enfermedades cardiovasculares, uso concomitante con otras medicaciones que prolonguen el intervalo QT, inhibidores del citocromo P-450, hipomagnesemia, hipocalcemia y mayores de 65 años, entre otros.

Cataldi $M$, Maurer $M$, Taglialatela $M$, Church MK. Cardiac safety of second-generation H1antihistamines when updosed in chronic spontaneous urticaria. Clin Exp Allergy 2019;49:1615-1623. 